\title{
Content Based Text Information Search and Retrieval in Document Images for Digital Library
}

\author{
A. Sakila ${ }^{1}$, S. Vijayarani ${ }^{2}$ \\ ${ }^{1} \mathrm{Ph}$. D Research Scholar, Department of Computer Science, Bharathiar University \\ Coimbatore, Tamilnadu, India \\ sakivani27@gmail.com \\ 2 Department of Computer Science, Bharathiar University \\ Coimbatore, Tamilnadu, India \\ vijimohan_2000@yahoo.com
}

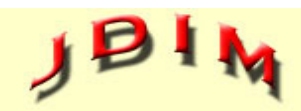

Journal of Digital

Information Management

\begin{abstract}
The main objective of this research work is to find the keywords in the captured/scanned print document images in the image database. Document images are becoming more popular in today's world and these are used in paperless offices and digital libraries. Information retrieval from the document images is a very challenging task. Hence, there is a need for developing searching strategies to find the required information from these document images as per user's needs, becomes very essential in nowadays. Traditionally Optical Character Recognition (OCR) tools are used for information retrieval from the document images, but it's not an efficient method. Word spotting is an inventive method for searching the document images and to retrieve relevant information without any conversion. In this work an algorithm Enhanced Dynamic Time Warping was proposed to for finding keywords from document images, it is based on word spotting technique. Different matching algorithms are made available for word spotting. Popular algorithms are Normalization Cross Correlation (NCC) and Dynamic Time Warping (DTW). In this work, we have compared the performance of these two existing algorithms with the proposed algorithm named as Enhanced Dynamic Time Warping algorithm (EDTW). Different image formats and different sizes of images are used for experimentation. From the results it is observed that the proposed algorithm has produced good results than an existing one.
\end{abstract}

Subject Categories and Descriptors

H.3.3 [Information Search and Retrieval]; H.3.7 [Digital Libraries]; I.7.5 [Document Capture]: Optical character recognition

General Terms: Digital Libraries, Information Retrieval,
Document Processing

Keywords: Document Image, Information Retrieval, Optical Character Recognition, Word spotting, Normalization Cross Correlation (NCC), Dynamic Time Warping (DTW)

Received: 10 October 2017, Revised 12 December 2017, Accepted 6 January 2018

DOI: $10.6025 / \mathrm{jdim} / 2018 / 16 / 3 / 136-151$

\section{Introduction}

Normally libraries and organizations are still handling large amount of printed materials such as books, bills, ledgers and questionnaires. They are excessive and voluminous; hence some libraries and organizations are slowly getting digitized [23]. Digitalized means paper documents can be converted into digital form by using digitization equipment's like scanners, digital cameras and mobile phones. This document images are used in various applications like educational libraries, historical document analysis, identification of scripts in different languages, recognize the handwritten images, signature verification, recognizing number plates in traffic signals and so on.

Document image analysis is used to recognize the text and graphics components in images. Two categories of document image analysis can be defined, they are textual processing and graphics processing [26]. In textual processing, significant components of the document images are skew detection, finding columns, paragraphs, text lines and words. Graphics processing deals with the 
non-textual line and symbol components like mathematical symbols, water marks and company logos. Information retrieval (IR) from document images has become a growing and challenging problem as compared with digital texts [26]. After converting the documents into digital form, each and every digitized document is stored in different image formats and it is very difficult to perform the search process, i.e. to search the particular keyword(s) in the digitized documents. This situation raises concerns about the development of new techniques and algorithms which can able to perform the search and retrieval process efficiently in document images. However information retrieval is concerned with content based document browsing, indexing and searching from a huge database of document images. The text retrieval from document images has made significant progress and addresses related information processing problems such as topic clustering and information filtering .

Currently, Optical Character Recognition (OCR) tools are used to perform the information retrieval task in a smaller level. Optical Character Recognition technique recognizes the text from the document images and is used to convert scanned image into editable text format [5]. Scanned images can easily extract that text with the help of different OCR Tools. Downsides of these tools are that it is very difficult to extract text because of different font sizes, styles, symbols, dark background and poor quality of document image [5]. Hence, necessitate an alternative approach for finding information to the large collections of document image database is needed. An inventive method to search the document images and retrieve relevant information without converting these images is done by using word spotting techniques which give better results than OCR tools [27].

The remaining portion of this paper is discussed as follows. Related Works are given in Section 2. Section 3 discusses the proposed method. Section 3 provides the detailed description about the document image processing. Section 4 gives the experimental results. Conclusion is given in Section 5.

\section{Related Works}

A. L. Kesidis et.al [1] proposed word spotting structure for accessing the content of historical machine-printed documents, without the use of an optical character recognition. The proposed methodology has evaluated the historical Modern Greek printed documents which was available during the seventeenth and eighteenth century. Character-based word model makes it possible to perform ASCIl queries, subject to the availability of a set of labeled prototype characters [1] [4]. To improve the efficiency of accessing and searching, natural language processing techniques have been addressed. Searching the document images using only a base word-form for locating all the corresponding inflected word-forms and a synonym dictionary that further facilitates access to the semantic context of documents [1] [4].
B. Gatos et.al [4]proposed a word spotting technique for searching keywords in historical printed documents. In this technique, word segmentation step is not required. Synthetic word images are created from keywords, and these images are compared to all the words in the digitized documents. Their proposed method is based on blockbased document image for performing template matching process which satisfied the invariance in terms of translation, rotation and scaling. It is required to constrain the applied matching process only on certain regions of interest [11].

Nikos Vassilopoulos, et.al [15] proposed a classification free word spotting system for printed historical document image and skipped many of the procedures of a common word spotting approach. Moreover, it does not include segmentation, feature extraction, clustering or classification stages [12]. Instead, it treats the queries as compact shapes and uses image processing techniques in order to localize a query in the document images [12]. This system was tested on a historical document collection and they obtained good results.

Sayantan Sarkar [20]has proposed a word spotting using character shape code for handling handwritten English document images. His proposed work is different from other word spotting techniques because it implements two levels of matching the search query [18] [2]. First one is based on word size and the next one is based on character shape code of the query. An index database is used to store the word segments which drastically reduce the search time.

Yue Lu, et.al [21] proposed a word spotting technique which is used to search a word portion in document images. Based on this work, one can estimate the relevancy between the document image word and the user-specified word. The present approach has the ability to search words in document images, whose portions match with the user-specified words. The user specified word and the word image extracted from documents are represented by two feature strings. Inexact string method is used to measure the similarity between the two feature strings. This approach has the ability to search words in document images, whose portions match with the user-specified words. But this approach cannot handle the italic word images due to the inherent shortcoming of the feature string used to represent the word image.

Partha Pratim Roy, et.al [25] has proposed a word spotting based information retrieval for medical prescriptions. A medical prescription is generally divided into two parts, a printed letterhead part and a handwritten part. To extract the information from document images, first they extracted the printed letterhead portion which contains the details about the doctor, i.e. name, qualification, etc and the handwritten part are detected from prescription using Hidden Markov Models (HMMs). An efficient MLP (MultiLayer Perceptron) based Tandem feature is proposed for improving the performance. 
K. Zagoris, et.al [30] proposed a novel technique for word spotting in hand written document images. It uses local proximity search without using any training data. They used both segmentation-based word spotting and segmentation-free word spotting methods. Four historical handwritten data sets are used for experiment analysis using standard evaluation measures. It is found that the proposed algorithm gives better performance than existing word spotting techniques.

Angelos P. Giotis, et.al [29] presented a comprehensive study on word spotting technique for various scripts or fonts. They analyzed a various word spotting techniques for match the keyword. Described the steps namely preprocessing, feature extraction, representation and similarity measures to retrieve information from the document images. Authors also discussed widely used datasets for word spotting, the state of the art in the most commonly used document image databases. Finally they conclude learning based word spotting technique gives better results than learning-free method. Learning-based methods without need for manually retrieve to searching the text queries.

By analyzing the literature, we come to know that some of the drawbacks of the existing techniques are,it does not match different font types, disparate font styles, and various font sizes and partially matching keywords. The main contribution of this research work is to find the keywords in the captured/scanned print document images in the image database using Enhanced DTW technique.
The proposed technique is able to retrieve keywords with different font types, disparate font styles, and various font sizes and partially matching keywords. Thus drawbacks of the existing techniques are overcome by the proposed technique.

\section{Proposed Method}

In the proposed method, features are preferred to capture the information of shape using OCR; Shape matching is performed at the local level with an enhanced DTW. The proposed system has five steps. They are preprocessing, edge detection, segmentation, optical character recognition (OCR) and matching. The architectural view of the proposed system is depicted in Figure 1.

\section{Document Image Processing}

\subsection{Preprocessing}

Pre-processing is the most important step for document image processing and it improves the searching and retrieval process. Paper documents can be converted into digital form by using digitization equipment. This digitization equipment's produced some noise like illumination and low resolution which usually degraded the quality of the document images [8]. Hence document images need to be pre-processed so as to improve the effectiveness of retrieval by reducing extraneous data and noise [19]. A color image then will be converted to a gray image before proceeding with the noise removal procedure.

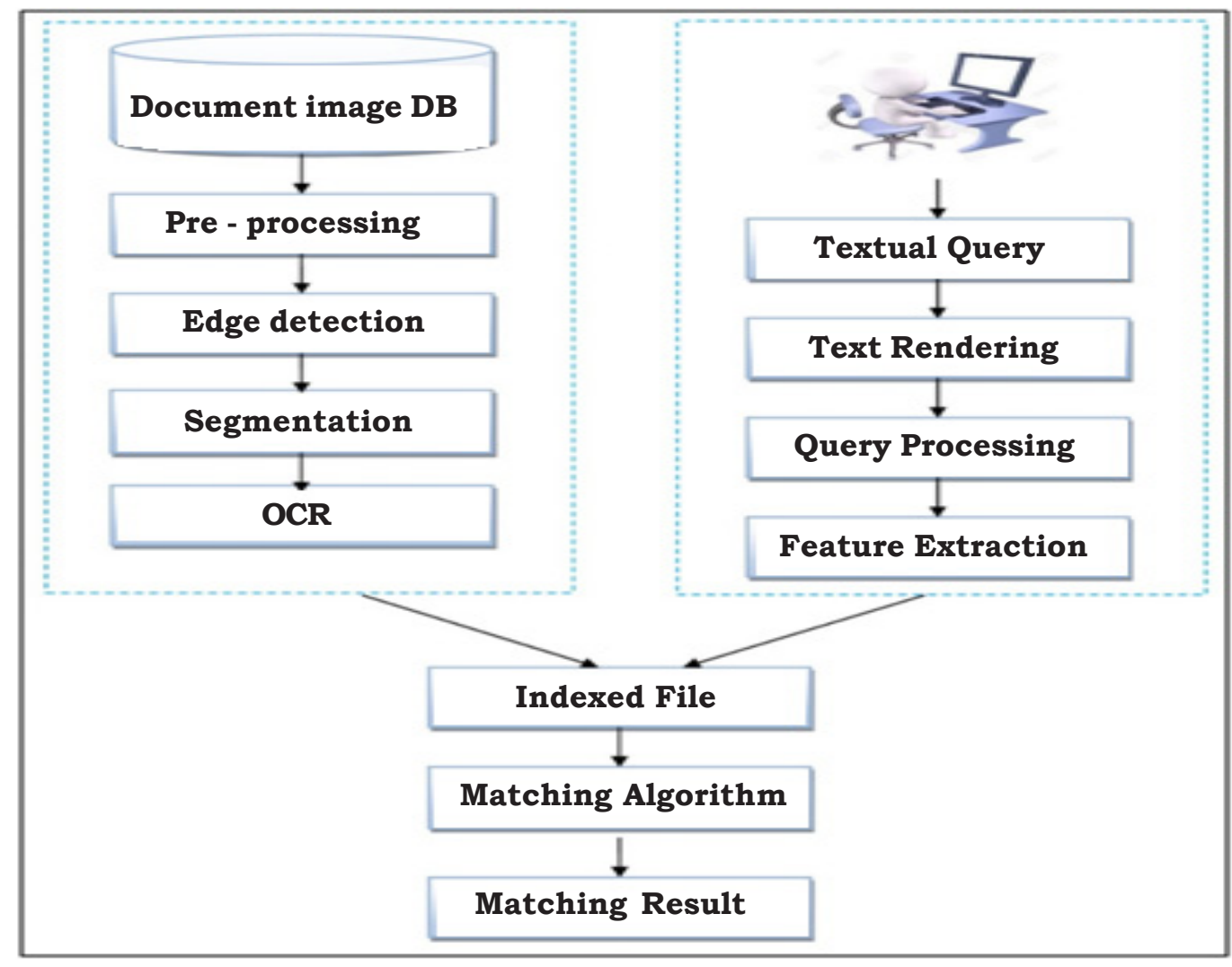

Figure 1. System Architecture 
The de-noised image is then converted to a binary image with suitable threshold. This stage involves preparing/ cleaning the data set by resolving problems like indeterminate data, unrelated fields, removal of distant points, format conversion, etc. In this research work, average filter, median filter, Gaussian filter, and wiener filter are used. From the experimental results is found that wiener filter gives better results than other filters, it removes salt and pepper and poison noise in the input image. Wiener filter takes less time and it gets high PSNR ratio compared with other filters.

\subsection{Edge Detection}

Edge detection is a significant task in the document image retrieval; it indicates the process of finding and locating sharp discontinuation of characters in the document images [6]. The main aim of edge detection is to discover the information concerning shapes and the reflectance or transmittance in an image. Some edge detection operators are available for extract the edge points in the document images. Each operator is designed to be sensitive to certain types of edges. In this work, the Roberts, Prewitt, Sobel and Canny edge detection techniques are used. For the performance of the experimental results, the Canny Edge detection method provides better results and takes less time and it gets high PSNR ratio than other edge detection techniques.

\subsection{Segmentation}

Segmentation is necessary for document images, it converts the text into lines, words and characters, and this is needed for performing optical character recognition based word spotting [24]. The main aim of image segmentation is the domain-independent partition of the image into a set of regions. This work has used three segmentation techniques to find the keyword in document images, they are line segmentation, word segmentation and character segmentation [10]. Each line in the document images may not be perfectly horizontal; they will not have so much of skew that there is no interline gap [9]. Hence the lines are aligned horizontally using line segmentation. With our observation, in order to do line segmentation in run-length compressed document, obtaining Horizontal Projection Profile is sufficient, provided that the document is skew free [16]. After segmenting, text documents are split into lines, carrying out word and character is segmented [13]. The word segmentation is used to separate the text region into the unit of lines and then finally into words [14]. Words are then split into characters using character segmentation. In the character segmentation, each character in the document image is shown in the bounding box. Figure 2 gives the input document image. Figure 3 shows the line segmented image. Figure 4 presents words are extracted from segmented line. Figure 5 displays character segmentation from segment word.

\section{Short Story Idea for Supercharge Your Writing. Feel free to add some romance to a supernatural story, or frame a family tale in a historical or dystopian setting. The key to using these short story ideas is an open, flexible mind. Use these prompts as springboards, and then follow your inspiration.}

Figure 2. Input image

\begin{tabular}{|l|}
\hline Short Story Idea for Supercharge Your Writing. Feel \\
\hline free to add some romance to a supernatural story, or \\
\hline frame a family tale in a historical or dystopian \\
\hline setting. The key to using these short story ideas is an \\
\hline open, flexible mind. Use these prompts as \\
\hline springboards, and then follow your inspiration. \\
\hline
\end{tabular}

Figure 3. Line Segmentation

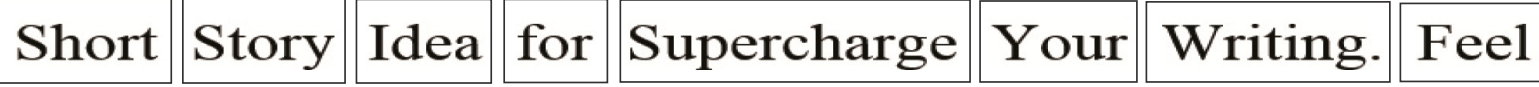

Figure 4. Word Line Segmentation 
Figure 5. Character Line Segmentation

\subsection{Optical Character Recognition (OCR)}

Optical Character Recognition (OCR) technique is used for text recognition in document images. Both OCR and Word spotting techniques are applied in this research work, to find the keywords accurately. Segmented character images are binarized into an array of $15 \times 15$, where black pixel representing 1 and white space representing 0 , given in Figure 6 . The binary format of each character is compared with the existing template. Binary format is divided into 5 tracks and each track subdivided into 8 sectors. Figure 7 shows the tracks and sectors representation.

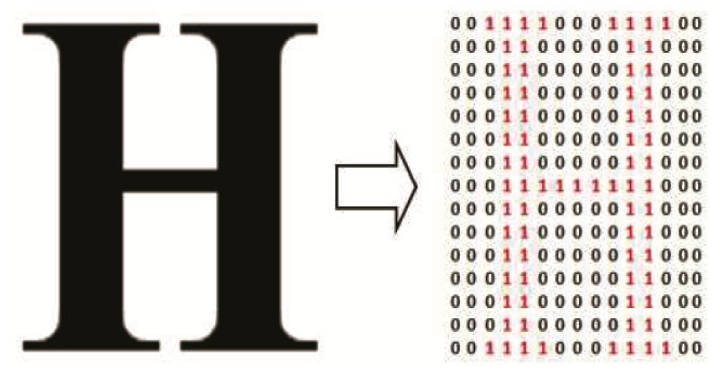

Figure 6. 15 X 15 Matrix

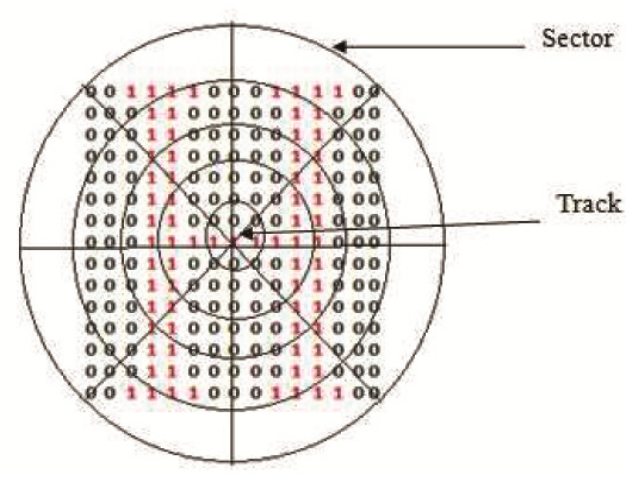

Figure 7. Representations of Tracks and Sectors

In the center of the matrix, there will be 1's in each inter- section of sector and track. The track - sector matrix is generated and matched with existing templates; it has each track-sector intersection value. If all the matrix values are found to match with the template value, the characters are identified in the document images. After identifying the character it will be stored in the indexed documents. This indexed document is the input for Enhanced DTW, which is used to find the keyword in document image in different font case (upper and lower case), font style (bold, italic, underline), font size (10-24) and variety of fonts (Arial, Book Antiqua, Calibri, Century, Garamond, High Tower Text, Times New Roman, etc...). Table 1 gives OCR Template Values for Alphabets

\subsection{Matching Algorithm}

The main task of matching algorithm is to compare the query features with the indexed features of the word images that are present in the document images [28]. In this section the proposed Enhanced Dynamic Time Warping (EDTW) technique was discussed with example. Also the existing techniques Normalized Cross Correlation (NCC) and Dynamic Time Warping (DTW) are illustrated. The performance of the proposed work is compared with these existing techniques.

\subsubsection{Normalized Cross Correlation (NCC)}

Normalized cross correlation (NCC) computes the similarity of two word images by aligning them against each other and then normalizing the result by the standard deviation of their features [3]. The concept of normalized correlation was developed to combat the effect of different illumination levels on image [2]. NCC computes the similarity between two word images if the length difference of the aligned features is less than the fixed threshold, it continues the process, until the aligned features fulfill the specified condition. The NCC value is confined in the range between -1 and 1 . The fixed threshold value is the feature length difference of the compared word images at each alignment. Based on the intensive experimentation mode this value is selected.

\begin{tabular}{|c|c|c|c|c|c|}
\hline $\begin{array}{c}\mathbf{A}, \mathbf{a} \\
1-10\end{array}$ & $\begin{array}{c}\mathbf{B}, \mathbf{b} \\
11-20\end{array}$ & $\begin{array}{c}\mathbf{C}, \mathbf{c} \\
21-30\end{array}$ & $\begin{array}{c}\mathbf{D}, \mathbf{d} \\
31-40\end{array}$ & $\begin{array}{c}\mathbf{E}, \mathbf{e} \\
41-50\end{array}$ & $\begin{array}{c}\mathbf{F , f} \\
51-60\end{array}$ \\
\hline $\begin{array}{c}\mathbf{G}, \mathbf{g} \\
61-70 \\
\end{array}$ & $\begin{array}{c}\mathbf{H}, \mathbf{h} \\
71-80 \\
\end{array}$ & $\begin{array}{c}\mathbf{I}, \mathbf{i} \\
81-90 \\
\end{array}$ & $\begin{array}{c}\mathbf{J}, \mathbf{j} \\
91-100 \\
\end{array}$ & $\begin{array}{c}\mathbf{K}, \mathbf{k} \\
101-110\end{array}$ & $\begin{array}{c}\mathbf{L}, \mathbf{l} \\
111-120 \\
\end{array}$ \\
\hline $\begin{array}{c}\mathbf{M}, \mathbf{m} \\
121-130\end{array}$ & $\begin{array}{c}\mathbf{N}, \mathbf{n} \\
131-140\end{array}$ & $\begin{array}{c}\mathbf{0}, \mathbf{0} \\
141-150\end{array}$ & $\begin{array}{c}\mathbf{P}, \mathbf{p} \\
151-160\end{array}$ & $\begin{array}{c}\mathbf{Q}, \mathbf{q} \\
161-170\end{array}$ & $\begin{array}{c}\mathbf{R}, \mathbf{r} \\
171-180\end{array}$ \\
\hline $\begin{array}{c}\mathbf{S}, \mathbf{S} \\
181-190\end{array}$ & $\begin{array}{c}\mathbf{T}, \mathbf{t} \\
191-200\end{array}$ & $\begin{array}{c}\mathbf{U}, \mathbf{u} \\
201-210\end{array}$ & $\begin{array}{c}\mathbf{V}, \mathbf{v} \\
211-220\end{array}$ & $\begin{array}{c}\mathbf{W}, \mathbf{w} \\
221-230\end{array}$ & $\begin{array}{c}\mathbf{X}, \mathbf{x} \\
231-240\end{array}$ \\
\hline \multicolumn{3}{|c|}{$\begin{array}{c}\mathbf{Y , y} \\
241-250\end{array}$} & \multicolumn{3}{|c|}{$\begin{array}{c}\mathbf{Z}, \mathbf{z} \\
251-260\end{array}$} \\
\hline
\end{tabular}

Table 1. OCR Template Values for Alphabets 


$$
\operatorname{Sim}\left(I_{1}, I_{2}\right)=\frac{\sum_{i=0}^{m} \Sigma_{j=0}^{n}\left(x_{i}-\text { mean }_{1}\right) *\left(x_{j}-\text { mean }_{2}\right)}{\delta I_{1} * \delta I_{2}}
$$

Where mean $I_{1}$ and mean $I_{2}$ refer to the mean and $I_{1}$ and $I_{2}$ refer to the standard deviation of the compared word images. A given feature vectors of word images: $=h_{1}, h_{2} \ldots h_{N}$ and $I=i_{1}, h i_{2} \ldots i_{N}$, Where and are maximum feature lengths. If the difference between each feature of the word image is greater than the threshold, then compute the normalized cross correlation, otherwise the same process is repeated.

\subsubsection{Dynamic Time Warping (DTW)}

Dynamic Time Warping (DTW) matching algorithm is used to compute a distance between two time sequences [7]. For a given two sequences, it finds the matching distance by computing the optimal alignment between the sample points [20]. The alignment is optimal in the sense that it minimizes a cumulative distance measure consisting of "local" distances between aligned samples [14]. The procedure is called Time Warping because it warps the time axes of the two time series in such a way that corresponding samples appear at the same location on a common time axis [17].

The DTW-distance between two time series $x_{1} \ldots x_{m}$ and $y_{1} \ldots y_{N}$ is $D(M, N)$ which calculate in a dynamic programming approach using

$$
D(i, j)=\min \left\{\begin{array}{c}
D(i, j-1) \\
D(i-1, j) \\
D(i-1, j-1)
\end{array}\right\}+d\left(x_{i}, y_{i}\right)
$$

\section{Pseudo code for Normalization Cross Correlation (NCC)}

//Read coordinates values from a document image

Step 1: For $j=0$ to $M$ (maximum height of the word image do)

Step2: For $i=0$ to $N$ (maximum width of the word image do)

Step3: If (pixel intensity of Image $(i, j)<=\delta$ ) then

Step 4: $\quad$ sum $=$ sum +1

Step5: Else

Step6: $\quad$ count $=$ count +1

Step 7: Endif

Step8: End for

Step9: If $(h i-I j<\gamma)$ then

Step10: $\operatorname{sim}\left(I_{1}, I_{2}\right)=\frac{\sum_{i=0}^{m} \sum_{j=0}^{n}\left(x_{i-\text { meanI }_{1}}\right) *\left(x_{j}-\text { meanI }_{2}\right)}{\delta I_{1} * \delta I_{2}}$

Step 11: Endif

Step12: Goto step 2 until the termination

Step 13: End for

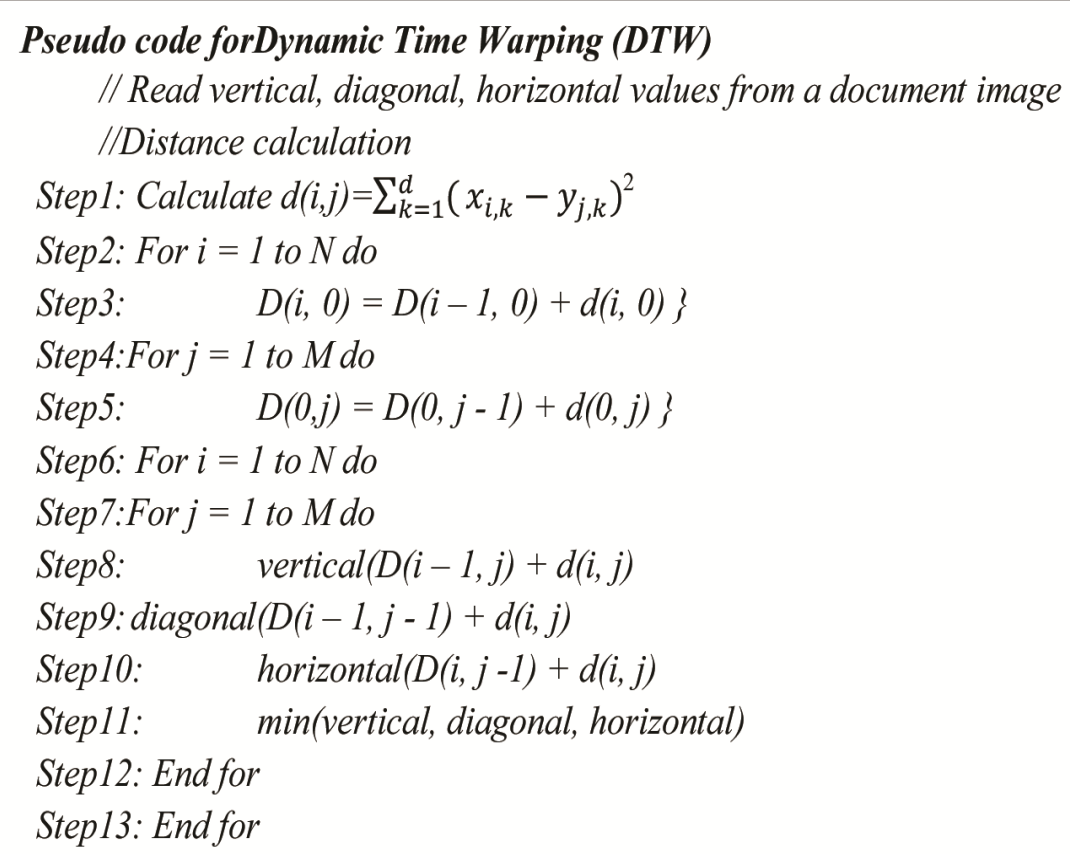


The particular choice of recurrence equation and "local" distance function $d\left(x_{i}, y_{i}\right)$ varies with the application. Using the given three values $D(i, j-1), D(i-1, j)$ and $D(i-1, j)$ in the calculation of $D(i, j)$ realizes a local continuity constraint which ensures smooth time warping [7]. DTW offers a more flexible way to compensate for these variations than linear scaling, in the matching algorithm, image columns are aligned and compared using DTW [20].

$$
d\left(x_{i}, y_{j}\right)=\sum_{k=1}^{d}\left(x_{i, k}-y_{j, k}\right)^{2}
$$

where $k$ is used to refer to the $k$-th dimension of $x_{i}$ and $y_{j}$. With this distance measure, it calculates the matching distance between two word images by comparing their features using DTW [22].

\subsubsection{Enhanced Dynamic Time Warping (EDTW)}

This research work has enhanced the existing Dynamic Time Warping algorithm, which is used to compute the similarity between two words in the document image. The performance is based on a DTW matching algorithm, by computing the optimal alignment " between the two points. An enhanced DTW algorithm is used to search the keyword in addition to find partial words in the document images. For partial matching we use $C$ be a coefficient function defined as

$$
C=\Sigma_{s=1}^{k} W_{s}
$$

Where $W$ is a keyword, $s$ is an index of the keyword and $k$ is initializing the index.

$$
p=C d\left(x_{i}, y_{j}\right)
$$

Where $p$ is a keyword matching against the next relevant words in the document image.

$$
p=\left\{\begin{array}{rr}
1 & C \vee d\left(x_{i}, y_{j}\right) \\
0 & \text { otherwise }
\end{array}\right.
$$

If $p=1$ the input keyword which matches the words in document image, otherwise no matches were found. Here example keyword "the" is partially matching in the words authenticate and then is given below.

$$
\left\{\begin{array}{c}
\text { Authenticate }=\frac{A u}{\left(s_{i}\right)} \frac{\text { the }}{\left(x_{i}-x_{j}\right)} \frac{\text { nticate }}{\left(s_{j}\right)} \\
\begin{array}{rl}
1 & 2 \\
\text { Then } & =\frac{\text { The }}{\left(x_{i}-x_{j}\right)} \frac{n}{\left(s_{j}\right)} \\
\text { the } & =\frac{\text { the }}{\left(x_{i}-x_{j}\right)} \\
2
\end{array} \\
=\text { Partial Matching (Keyword is: the) }
\end{array}\right.
$$

\section{Features of Proposed EDTW}

The proposed algorithm find the keywords in the document images. Content available in the document images may have, different font types, different Font case, disparate font styles and various font sizes.

- The proposed algorithm finds the keywords with different font types like Arial, Book Antiqua, Calibri, Century, Garamond, High Tower Text, Times New Roman, etc.

- It search the keyword in both upper case and lower case.

- It retrieve keywords even if it appears in disparate font styles bold, italic, underline or normal.

$$
\begin{aligned}
& \text { Pseudo code for Enhanced Dynamic Time Warping (EDTW) } \\
& \text { Step 1: Calculate } d(i, j)=\sum_{k=1}^{d}\left(x_{i, k}-y_{j, k}\right)^{2} \\
& \text { Step2: For } i=1 \text { to } N \text { do } \\
& \text { Step3: } \quad D(i, 0)=D(i-1,0)+d(i, 0) \\
& \text { Step4: End for } \\
& \text { Step5: For } j=1 \text { to } M d o \\
& \text { Step6: } \quad D(0, j)=D(0, j-1)+d(0, j) \\
& \text { Step 7: End for } \\
& \text { Step8: For } i=1 \text { to } N \text { do } \\
& \text { Step9: For } j=1 \text { to } M \text { do } \\
& \text { Step10: } \operatorname{vertical}(D(i-1, j)+d(i, j) \\
& \text { Step 11: } \quad \operatorname{diagonal}(D(i-1, j-1)+d(i, j) \\
& \text { Step 12: horizontal }(D(i, j-1)+d(i, j) \\
& \text { Step 13: min(vertical, diagonal, horizontal) } \\
& \text { Step 14: End for } \\
& \text { Step15: } C=\sum_{s=1}^{k} w_{s} \\
& \text { Step 16: } p=C d\left(x_{i}, y_{j}\right) \\
& \text { Step17: } p=\left\{\begin{array}{cc}
1 & C \vee d\left(x_{i}, y_{j}\right) \\
0 & \text { otherwise }
\end{array}\right. \\
& \text { Step18: End for }
\end{aligned}
$$$$
\text { // read partial matching from a document image based on DTW }
$$ 
- The proposed algorithm finds the keywords with various font sizes.

- It finds partial matching keyword.

- It can search multiple keywords.

\subsection{Textual Query and Text Rendering}

Whenever, a user provides a textual query word, the query word is first rendered. Text rendering converts the user's query into equivalent word image. i.e. Text query is converted into query image and this rendering process is depicted in Figure 8. After rendering the keyword, the keywords are partially matched in the input document image. Figure 9 displays the keyword "idea" which matches the OCR database.

\section{Experimental Results}

This research work is based on word spotting technique for finding keywords from document images. To perform intensive experimentation, scanned and captured document images are used. This research work has used more than two hundred different document image formats (JPG, png, bmp and tiff) with different sizes for experimentation.

\section{Matching Algorithm}

This research work has compared two existing algorithms namely Normalized Cross Correlation (NCC), Dynamic Time Warping (DTW) and one proposed algorithm named as Enhanced Dynamic Time Warping (EDTW) algorithm.

\begin{tabular}{|c|c|c|c|}
\hline Rendering & Rendering & Rendering & Rendering \\
\hline Test $\longrightarrow$ test & plant $\longrightarrow$ plant & finger $\longrightarrow$ finger & HOCKEY $\longrightarrow$ HOCKEY \\
\hline Test/TEST/test & Plant/PLANT/plant & $\begin{array}{l}\text { Finger/FINGER/ } \\
\text { finger }\end{array}$ & $\begin{array}{c}\text { Hockey/HOCKEY/ } \\
\text { hockey }\end{array}$ \\
\hline $\begin{array}{c}\text { Testing/TESTING/ } \\
\text { testing }\end{array}$ & $\begin{array}{c}\text { Planting/PLANTING/ } \\
\text { planting }\end{array}$ & $\begin{array}{l}\text { Fingerprint/ } \\
\text { FINGERPRINT/ } \\
\text { fingerprint }\end{array}$ & \\
\hline $\begin{array}{c}\text { Tested/TESTED/ } \\
\text { tested }\end{array}$ & & & \\
\hline
\end{tabular}

Figure 8. Text Rendering and Partial Matching

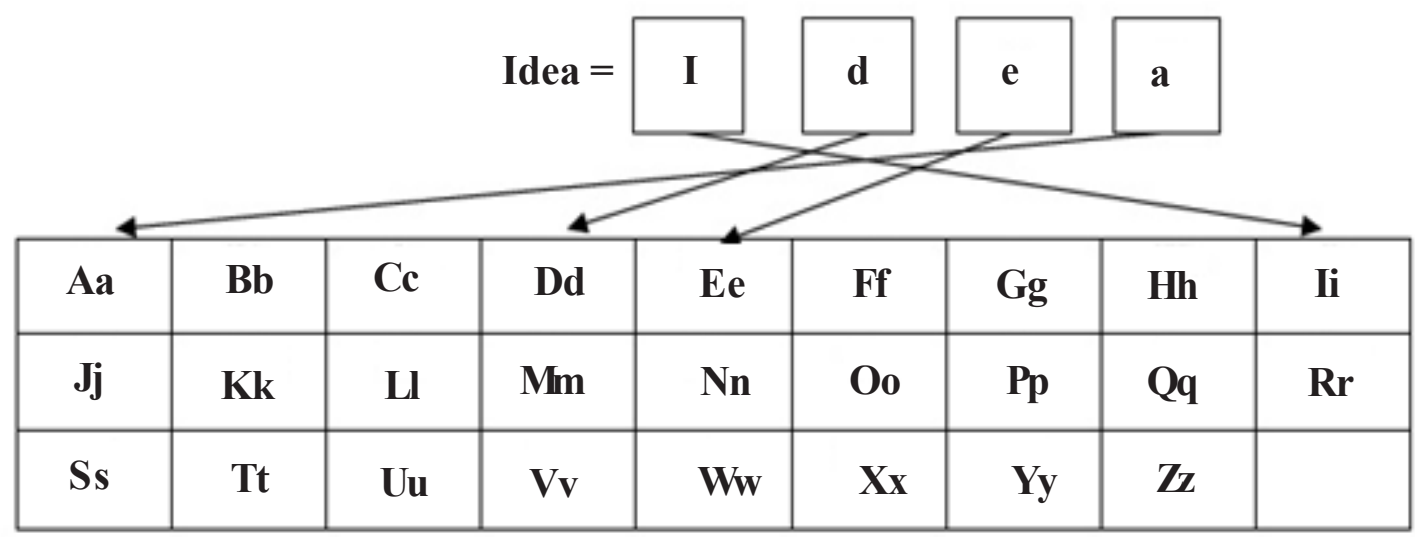

Figure 9. Keyword matches in OCR Database

\subsection{Query Preprocessing and Feature Extraction}

After the rendering task, the query image is binarized and its features are extracted based on similarity measure which finds similar index terms. Accordingly, if the computed similarity values fulfill the minimum threshold á, document images with index terms are retrieved and ranked according to their relevance for the given query. Then the similarity between the query and the word image in the index file is computed and document images are returned according to their order of relevance.
Experimental results proved that Enhanced Dynamic Time Warping (EDTW) algorithms finds keywords in document images correctly than existing algorithms.

\subsection{Accuracy Measure for Matching Algorithms}

The precision, recall and F-Score measures have been used to evaluate the performance of the proposed and the existing techniques. Figure 10 represents the input image. Table 2 shows the performance measures for Normalized cross correlation, Dynamic time warping and Enhanced 


\section{SYSTEM TESTING}

\section{White-box testing}

White box testing focus on the program control structure. Test cases are derived to ensure that all statements in the program have been executed at least once during testing and that all logical conditions have been executed.

\section{Block-box testing}

Black box testing is designed to validate functional requirements without regard to the internal workings of a program. Black box testing mainly focuses on the information domain of the software, deriving test cases by partitioning input and output in a manner that provides through test coverage. Incorrect and missing functions, interface errors, errors in data structures, error in functional logic are the errors falling in this category.

\section{Unit testing}

Unit testing is essential for the verification of the code produced during the coding phase and hence the goal is to test the internal logic of the modules. Using the detailed design description as a guide, important paths are tested to uncover errors within the boundary of the modules. These tests were carried out during the programming stage itself.

Figure 10. Input image

\begin{tabular}{|c|c|c|c|c|c|c|c|c|c|c|}
\hline \multirow[t]{2}{*}{ Item } & \multirow[t]{2}{*}{ Type } & \multicolumn{3}{|c|}{$\begin{array}{l}\text { Normalization Cross } \\
\text { Correlation (NCC) } \\
\end{array}$} & \multicolumn{3}{|c|}{$\begin{array}{l}\text { Dynamic Time Warping } \\
\text { (DTW) }\end{array}$} & \multicolumn{3}{|c|}{ Enhanced DTW } \\
\hline & & Rec. & Pre. & Fsc. & Rec. & Pre. & Fsc. & Rec. & Pre. & Fsc. \\
\hline \multirow{2}{*}{$\begin{array}{l}\text { Font } \\
\text { Case }\end{array}$} & Upper Case & 50.80 & 61.53 & 61.53 & 84.68 & 88.48 & 88.12 & 99.31 & 100 & 98.56 \\
\hline & Lower Case & 62.51 & 72.33 & 71.42 & 97.67 & 98.52 & 97.15 & 100 & 100 & 100 \\
\hline \multirow{4}{*}{$\begin{array}{l}\text { Font } \\
\text { Size }\end{array}$} & 11 & 63.52 & 68.21 & 67.53 & 91.12 & 93.26 & 92.98 & 98.97 & 100 & 100 \\
\hline & 12 & 69.93 & 71.93 & 71.49 & 92.32 & 96.51 & 95.33 & 100 & 100 & 100 \\
\hline & 14 & 72.82 & 75.18 & 74.92 & 93.83 & 97.86 & 95.91 & 100 & 100 & 100 \\
\hline & 18 & 12.32 & 14.32 & 14.9 & 77.52 & 79.82 & 79.34 & 89.65 & 93.41 & 96.72 \\
\hline \multirow{4}{*}{$\begin{array}{l}\text { Font } \\
\text { Style }\end{array}$} & Normal & 75.28 & 78.28 & 77.98 & 92.72 & 95.72 & 89.53 & 100 & 100 & 100 \\
\hline & Bold & 64.25 & 69.55 & 69.17 & 82.96 & 86.51 & 85.31 & 95.30 & 100 & 97.12 \\
\hline & Italics & 0 & 0 & 0 & 58.86 & 61.56 & 60.25 & 91.78 & 98 & 92.60 \\
\hline & Underline & 0 & 0 & 0 & 53.48 & 55.36 & 54.21 & 83.96 & 96.41 & 88.64 \\
\hline \multirow{5}{*}{$\begin{array}{c}\text { Font } \\
\text { Types }\end{array}$} & Arial & 29.43 & 32.93 & 44.39 & 39.87 & 81.87 & 83.58 & 94.45 & 96.29 & 96.87 \\
\hline & $\begin{array}{c}\text { Book } \\
\text { Antique }\end{array}$ & 45.98 & 42.34 & 52.94 & 60.25 & 84.21 & 86.46 & 97.12 & 98.84 & 100 \\
\hline & Calibri & 19.34 & 18.29 & 32.20 & 32.34 & 78.94 & 79.65 & 91.98 & 99.23 & 98.45 \\
\hline & $\begin{array}{l}\text { High Tower } \\
\text { Text }\end{array}$ & 33.98 & 31.84 & 39.67 & 47.04 & 81.83 & 84.62 & 96.47 & 100 & 100 \\
\hline & $\begin{array}{l}\text { Time New } \\
\text { Roman }\end{array}$ & 62.34 & 60.54 & 68.38 & 74.21 & 91.25 & 92.89 & 100 & 100 & 100 \\
\hline
\end{tabular}

Table 2. Performance Measures for Matching Algorithms

DTW for the input image shown in figure 10.

Figure 11shows the Font Case accuracy measures for matching algorithms, for the performance measure enhanced DTW performs better than existing algorithms.

Figure 12 displays the different font size $(11,12,14$ and18) accuracy measures for matching algorithms, for the performance measure enhanced DTW performs better than existing algorithms.
Figure 13 displays the different font style (Normal, Bold, Italics, Underline) accuracy measures for matching algorithms, for the performance measure enhanced DTW performs better than existing algorithms.

Figure 14 displays the different font types (Arial, Book Antiqua, Calibri, Century, Garamond, High Tower Text, Times New Roman, etc...) accuracy measures for matching algorithms, for the performance measure enhanced DTW performs better than existing algorithms. 


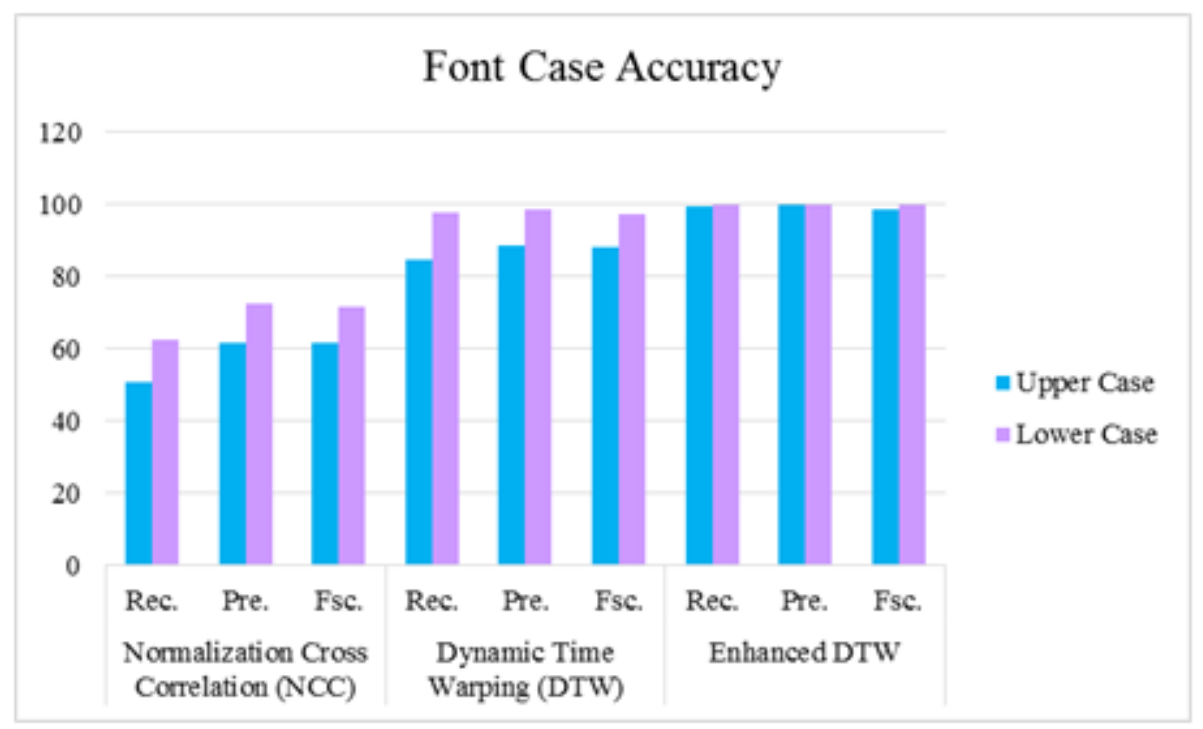

Figure 11. Font Case accuracy

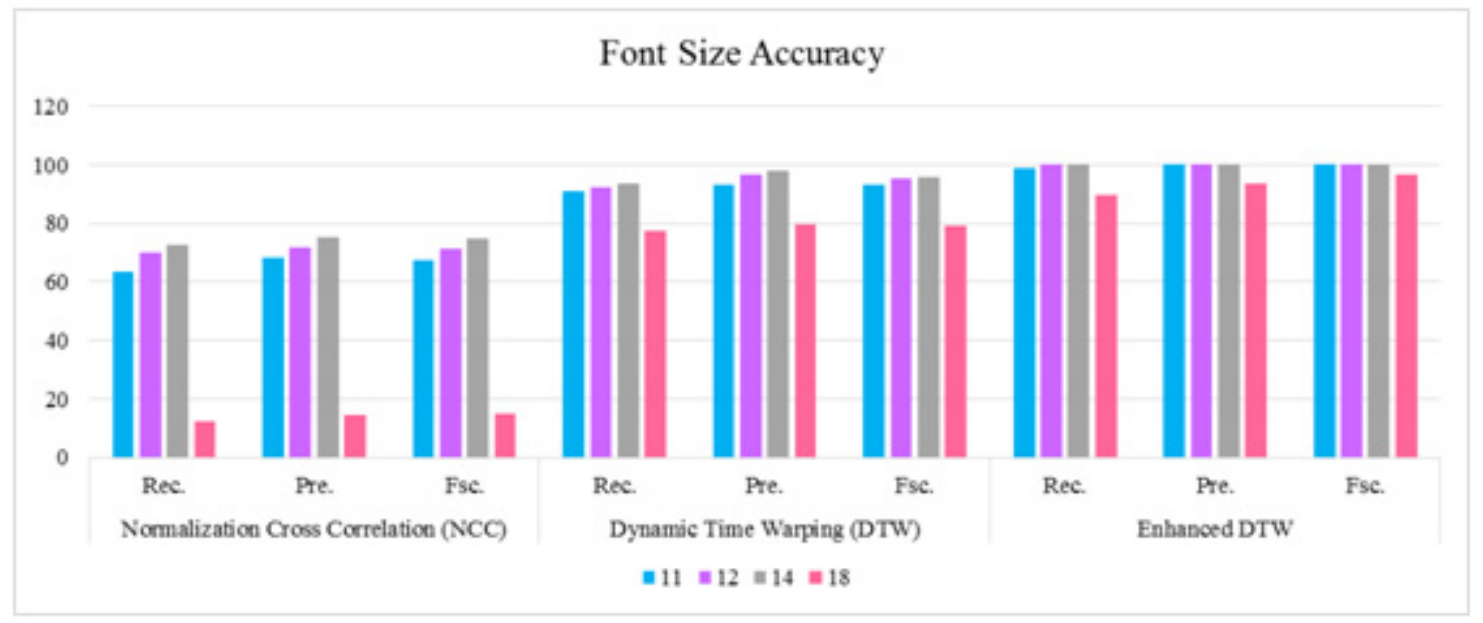

Figure 12. Font Size accuracy

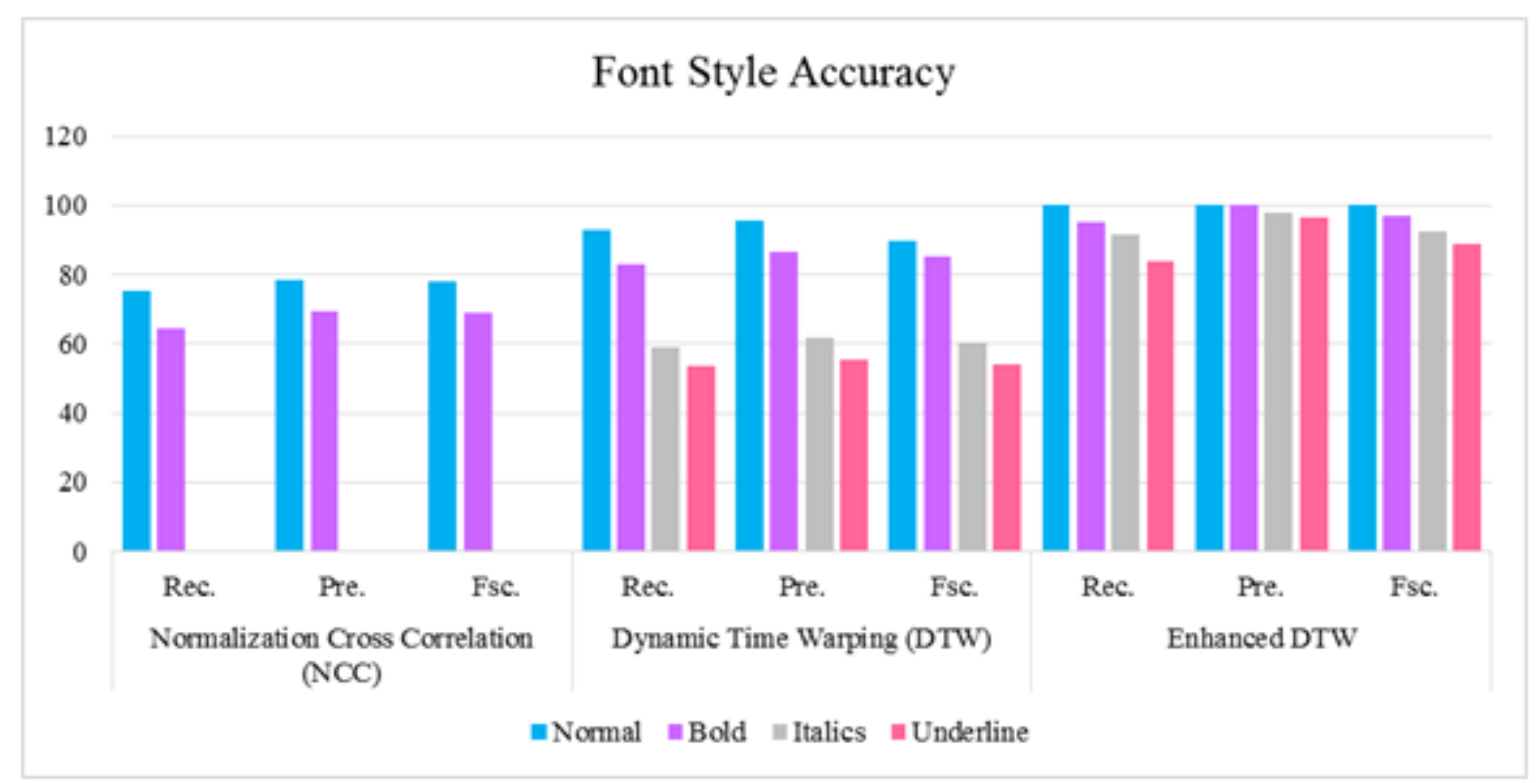

Figure 13. Font style Accuracy 


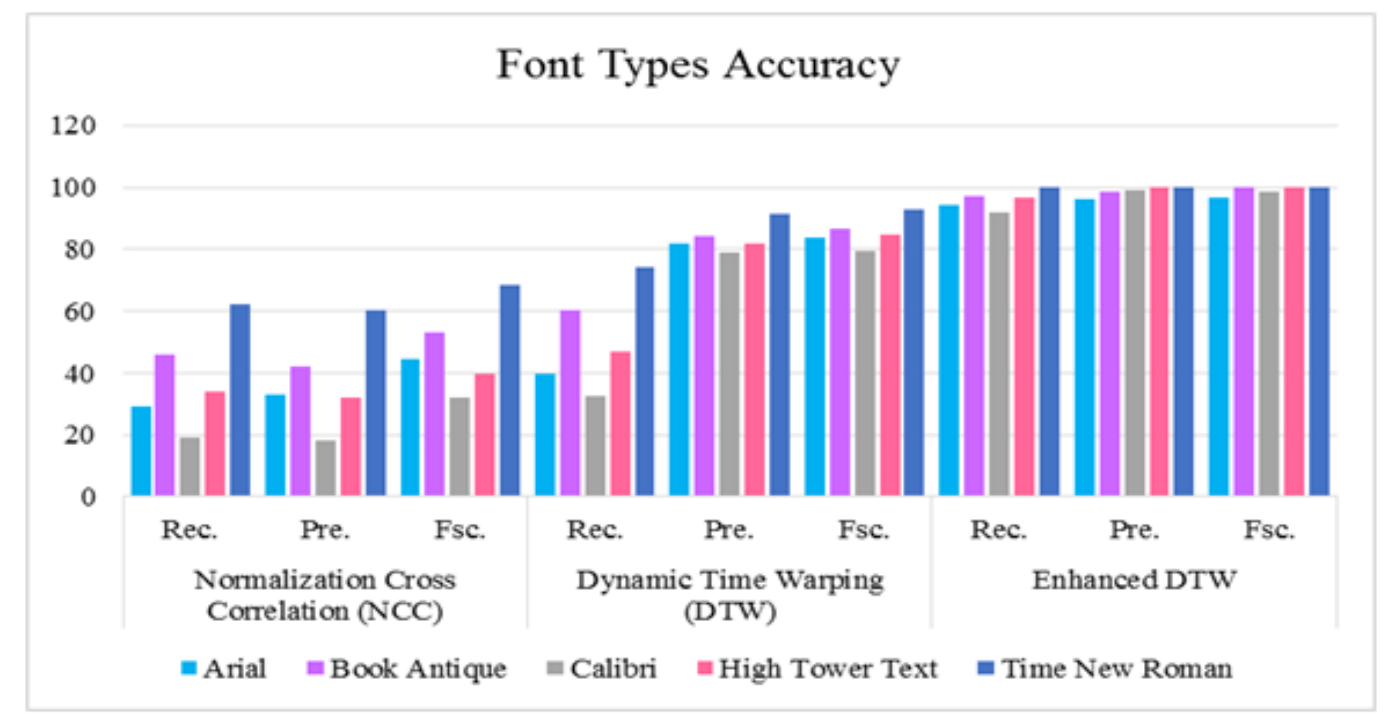

Figure 14. Font Types Accuracy

\subsection{Execution Time for Matching Algorithms}

Table 4 gives the execution time required for Normalized cross correlation, Dynamic time warping and Enhanced DTW. From figure 15, it is found that Enhanced DTW algorithm has required minimum execution time compared with normalized cross correlation and dynamic time warping algorithms.

\subsection{Word Spotting Results of EDTW}

Enhanced DTW results are given from figure 16 to figure 21. Figure 16 shows the keyword "plant" which matches the various fonts is Arial, Book Antiqua, Calibri, High Tower Text, Times New Roman. Figure 17 represents the keyword "test" which matches the different font styles such as normal, bold, italics and underline characters everywhere in the document image. Figure 18 displays the uppercase keyword "HOCKEY" is matches both uppercase, lowercase and sentence case letters. Figure 19 shows the multiple keywords are matched in the document image; the keyword is "distributed operating system". Figure 20 gives result for the keyword "finger" in the document image database. It was found that the word "finger" appears in six images and all the six images are listed in right side panel of the figure. Figure 21 represent the keyword "Child" which matches cursive fonts.

\begin{tabular}{|c|c|}
\hline Matching algorithm & $\begin{array}{c}\text { Execution Time } \\
\text { (Milliseconds) }\end{array}$ \\
\hline Normalized Cross Correlation (NCC) & 34480 \\
\hline Dynamic Time Warping (DTW) & 33850 \\
\hline Enhanced DTW & 19300 \\
\hline
\end{tabular}

Table 4. Execution Time for matching algorithm

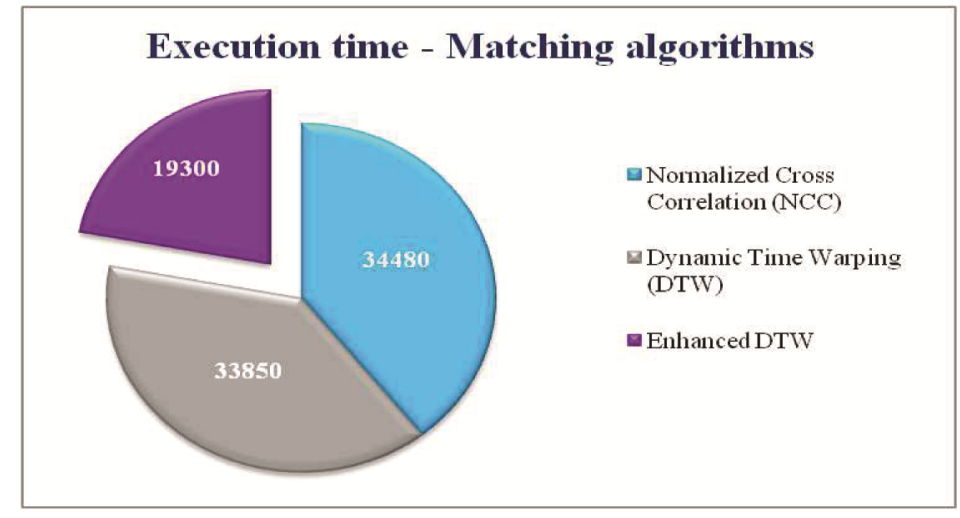

Figure 15. Execution Time 


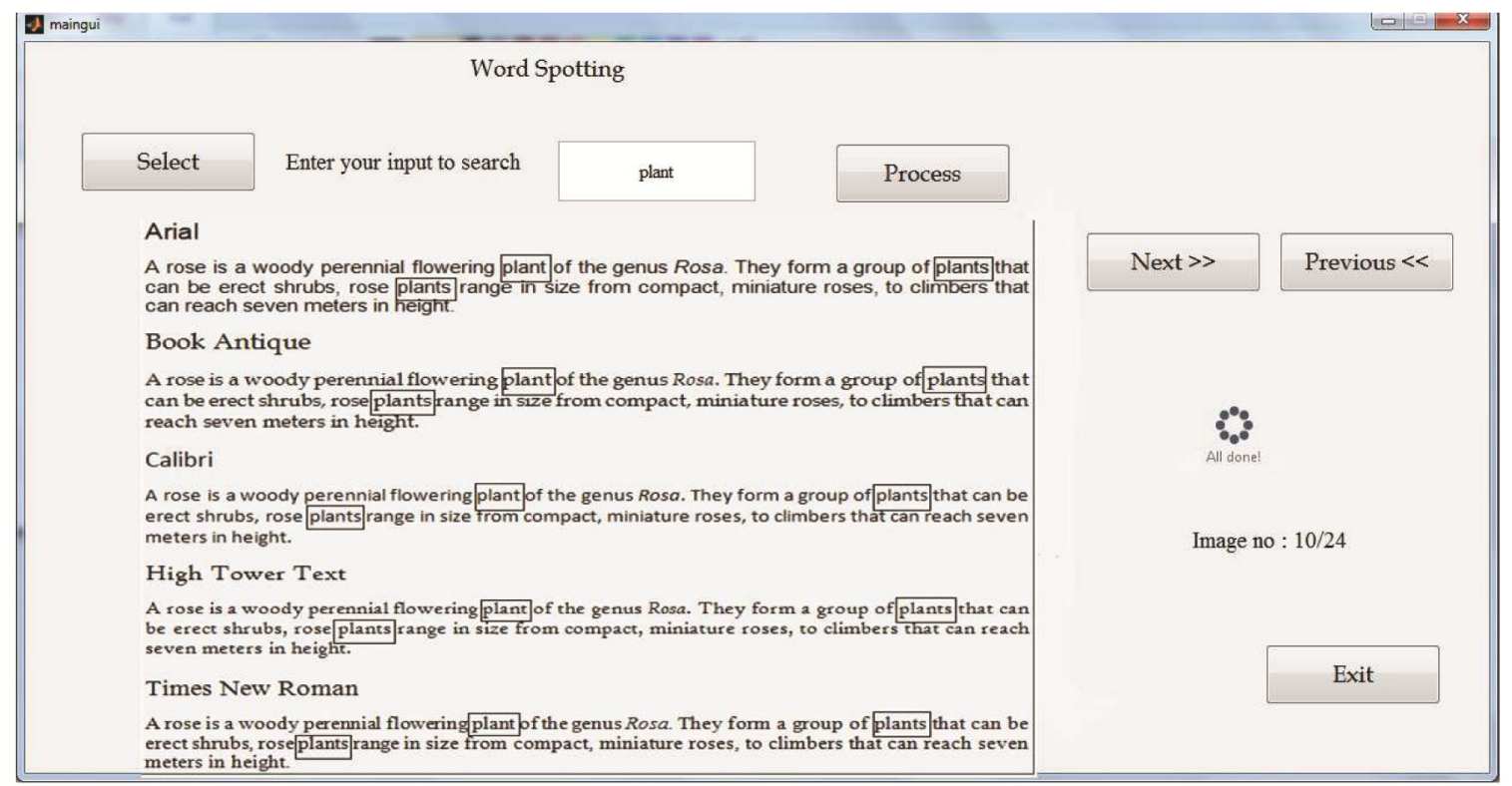

Figure 16. Search result by Enhanced DTW algorithm:Search query "plant"which matches the various fonts is Arial, Book Antiqua, Calibri, High Tower Text, Times New Roman

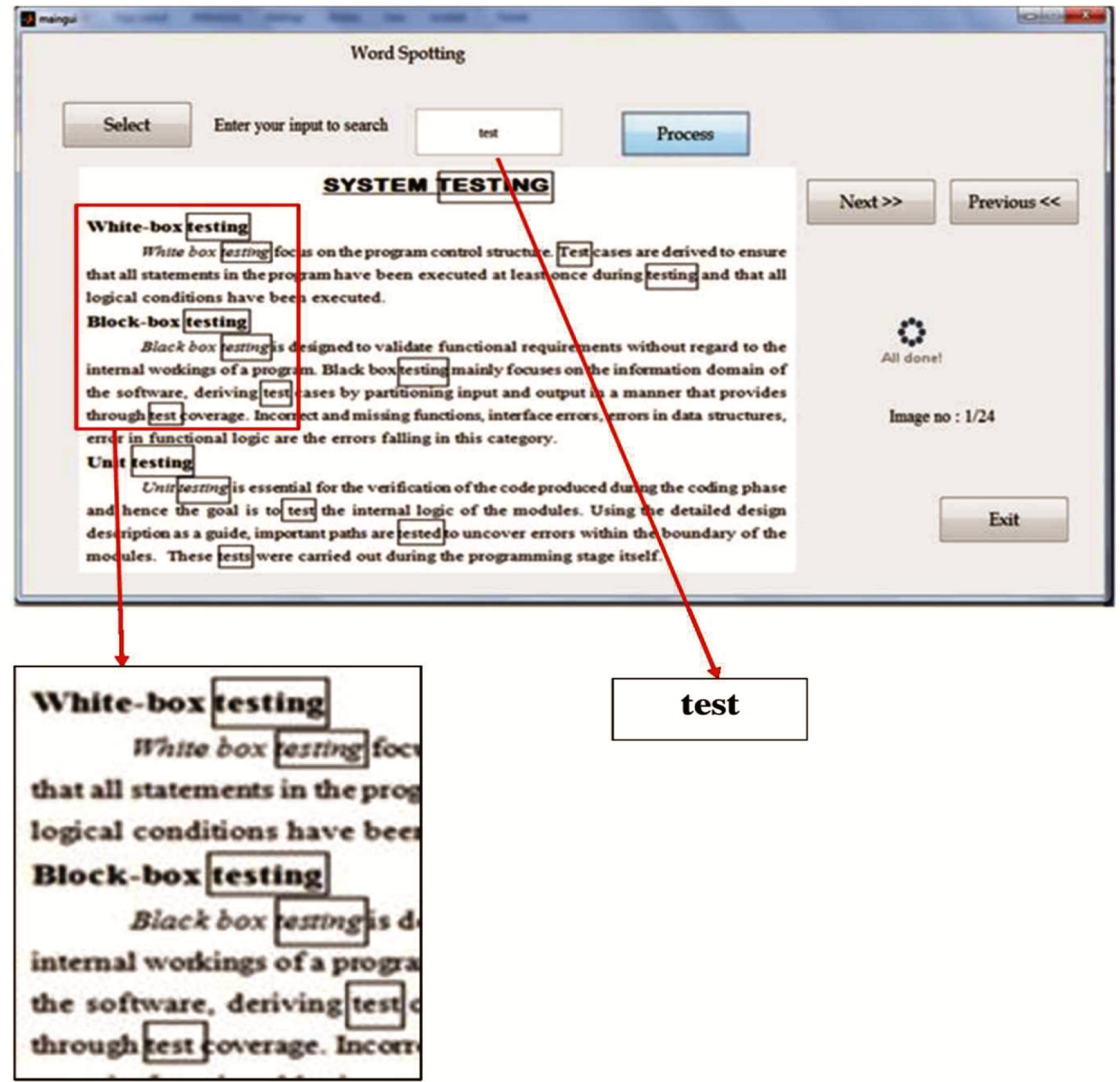

Figure 17. Search result by Enhanced DTW algorithm: Search query "test" which matches the different font styles such as normal, bold, italics and underline characters everywhere in the document image 


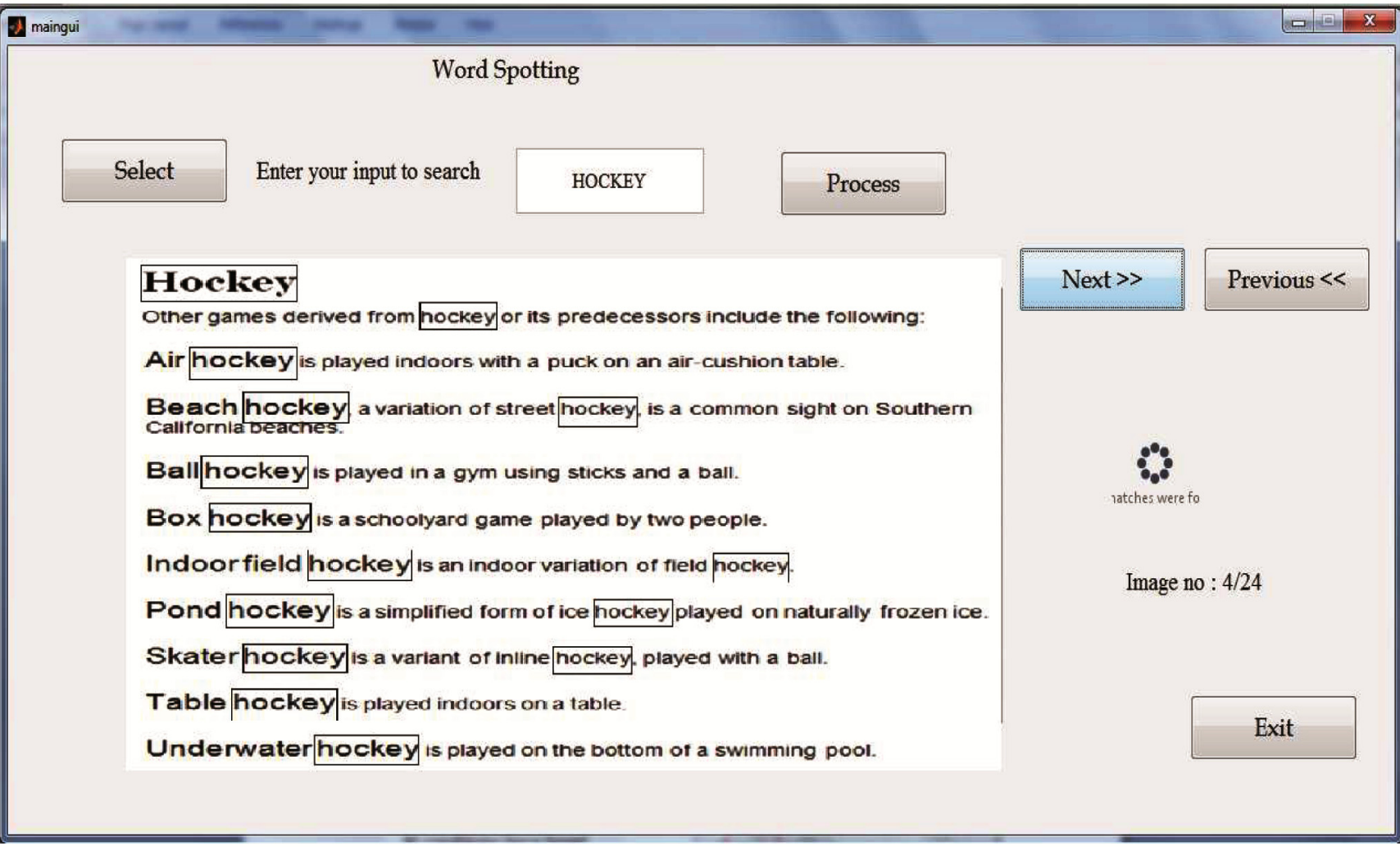

Figure 18. Enhanced DTW algorithm: Search query "HOCKEY" is matches both uppercase, lowercase and sentence case letters

\section{Word Spotting}

Select

Enter your input to search

distributed operating system

Process

A distributed operating system is a software over a collection of independent, networked, communicating, and physically separate computational nodes. Each individual node holds a specific software subset of the global aggregate operating system. A distributed operating system provides the essential services and functionality required of an $O S$ but adds attributes and particular configurations to allow it to support additional requirements such es increased scale and availability. To a user, a distributed operating system works in a manner similar to a single-node, monclithic operating system.

In a distributed operating system, the exceptional degree of

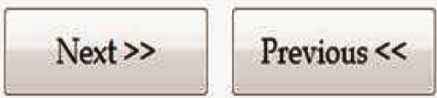
inherent complexity could easily render the entire system an anathema to any user. As such, the logical price of realizing a distributed operating system must be calculated in terms of overcoming vast amounts of comp-exity in many areas, and on many levels.

Figure 19. Search result by Enhanced DTW algorithm: Search multiple keyword "distributed operating system" 


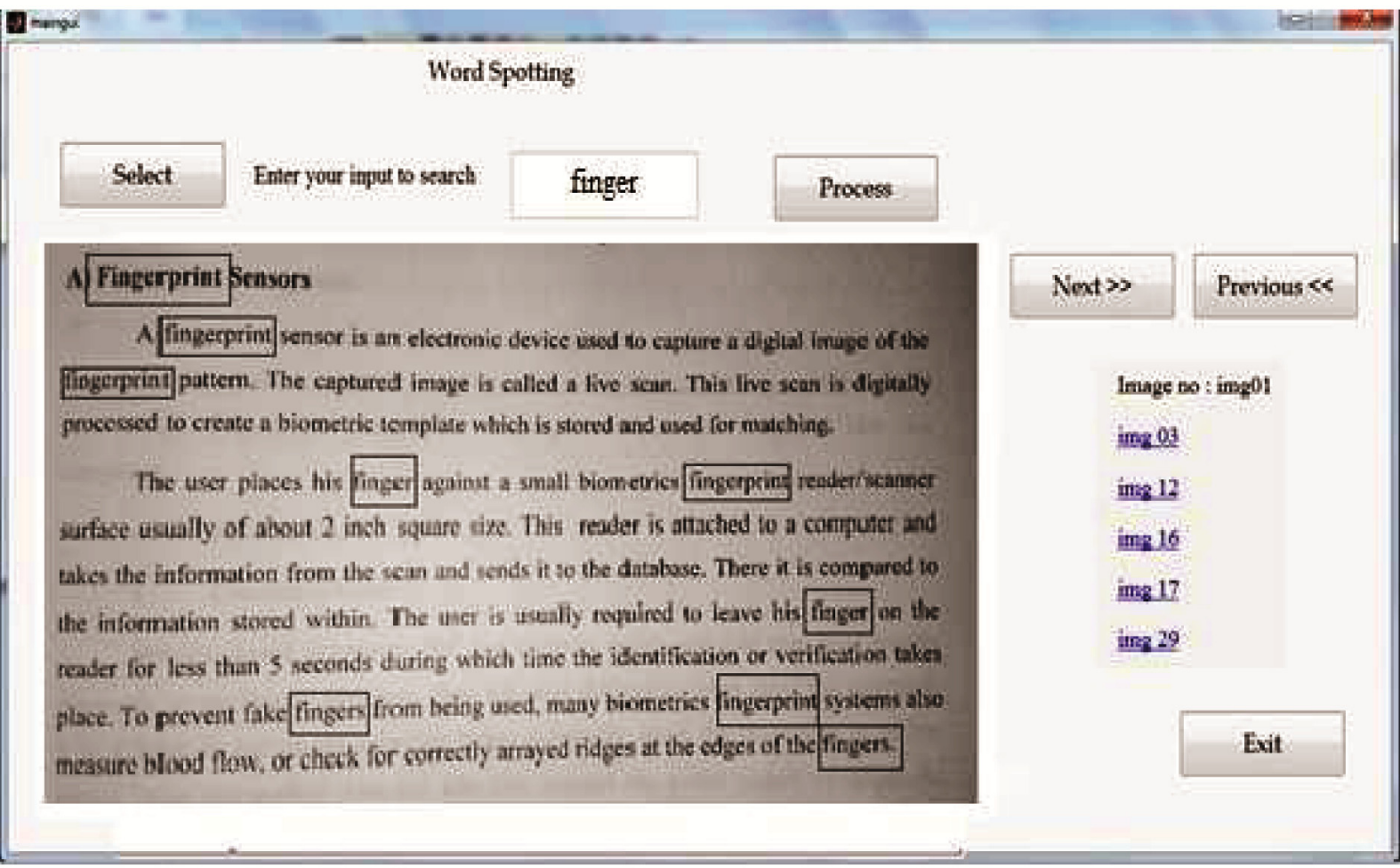

Figure 20. Enhanced DTW algorithm: Search query "finger" appears in six images and all the six images are listed in right side panel of the figure

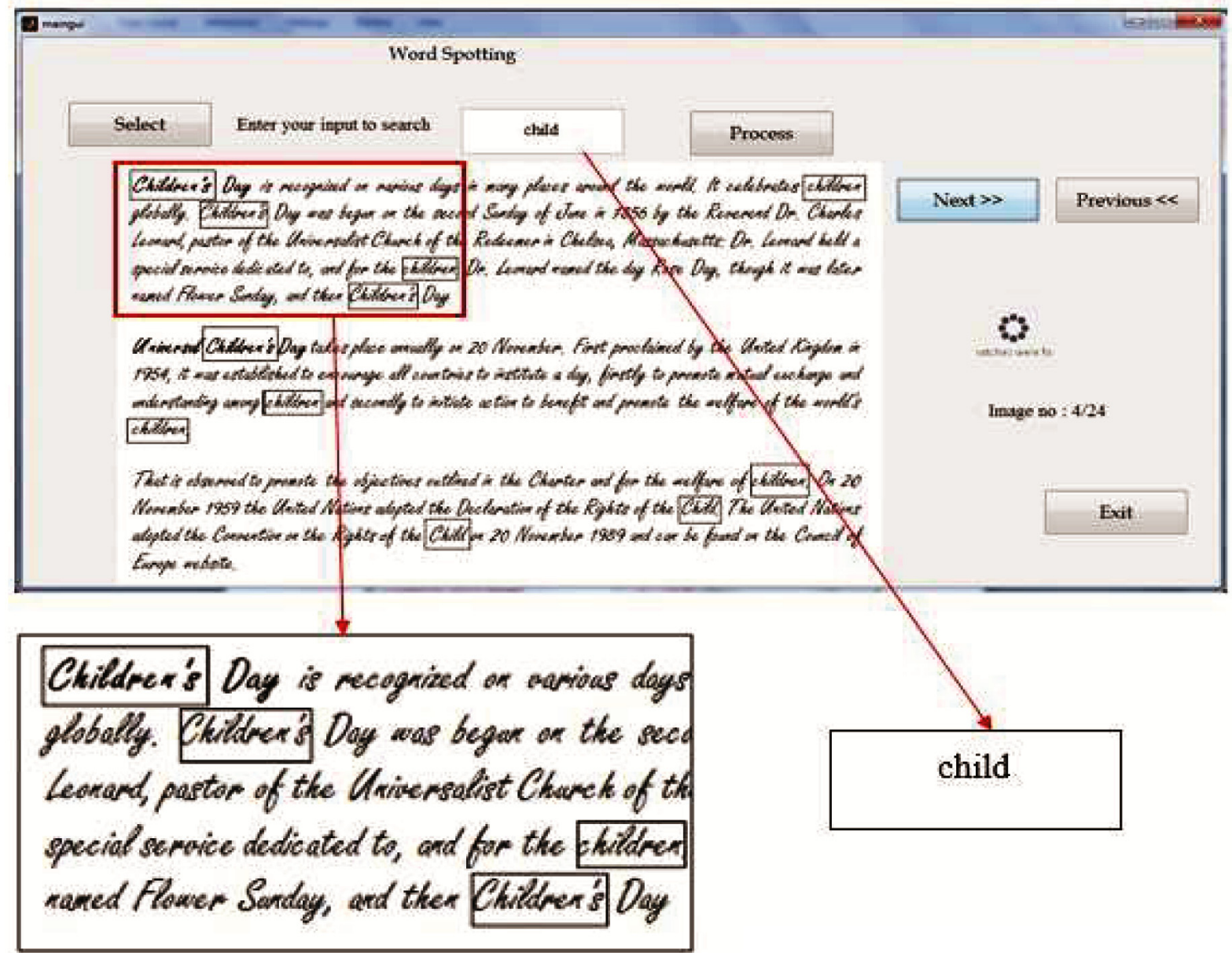

Figure 21. Enhanced DTW algorithm: Search query "Child" which matches cursive fonts 


\section{Conclusion and Future Work}

In this research work Enhanced DTW matching algorithm is proposed for finding user specified keyword in the document images. It is compared with Dynamic time warping (DTW) and Normalized Cross-Correlation (NCC). Based on the experimental results, it is found that the Enhanced DTW is better than existing algorithms; it supports different font size and styles. The proposed algorithm handles word variants and different font types, font sizes, font styles and partial match's keyword in the scanned document images. In addition, the enhanced DTW searches multiple keywords in the given document image. In future, this research work will be extended for handling large amount of quality and non-quality document images.

\section{Reference}

[1] Kesidis, A. L., Galiotou, E., Gatos, B., Pratikakis, I. (2010). A word spotting framework for historical machineprinted documents, IJDAR, Springer-Verlag,

[2] Lawrence Spitz, A. (1995). Character Shape Code for word spotting in document images, D. Dori and A. Bruckstein, eds., World Scientific, Singapore.

[3] Aldali., Nureddin, Ali. F., Jungang, Miao. (2015). Comparisons between BGI and Wiener Filter Method In Resolution Enhancement of Simulated Image, Proceedings of 44th IRF International Conference, 29th (November), Pune, India.

[4] Gatos, Pratikakis, I. (2009). Segmentation-free Word Spotting in Historical Printed Documents, 10th International Conference on Document Analysis and Recognition, IEEE 236, p.271-275.

[5] Vijayarani, S., Sakila, A. (2015). Performance Comparison of OCR Tools, International Journal of UbiComp (IJU), 6(3) (July)19-30.

[6] Vijayarani, S., Sakila, A. (2016). A Performance Comparison of Edge Detection Techniques for Printed and Handwritten Document Image, International Journal of Innovative Research in Computer and Communication Engineering, 4(5) (May).

[7] Nagendar, G., Jawahar, C. V. (2015). Efficient Word Image Retrieval using Fast DTW Distance, In: International Conference on Document Analysis and Recognition (ICDAR 2015).

[8] Garima, Ashraf, Javed. (2013). An advanced filter for image enhancement and restoration, International Journal of Innovative Research and Studies (IJIRS), 2 (6) (June).

[9] https://www.researchgate.net/publication/261253745_ Extraction_of_Line_Word_Character_Segments_Directly_ from_Run_Length_Compressed_Printed_Text_Documents
[10] Yan, Hum., Chai, Eko., Supriyanto., Lai., Wee, Khin. MRI Brain Tumor Image Segmentation using RegionBased Active Contour Model, Latest Trends in Applied Computational Science.

[11] Cao, Huaigu., Venu, Govindaraju. (2006). Templatefree word spotting in low-quality manuscripts, September 30, 2006. (http://citeseerx.ist.psu.edu/viewdoc/download? doi=10.1.1.92.9015\&rep=rep1\&type=pdf)

[12]Zagoris, Konstrantinos., Ergina., Kavallieratou., Papamarkos, Nikos. (2010). A Document image retrival system, Engineering Application of Artificial Intelligence, 23 (6) (September).

[13] Manish, T., Wanjari, Keshao, D., Kalaskar, Mahendra, P., Dhore. (2015). Document Image Segmentation Using Edge Detection Method, International Journal of Computer \& Mathematical Sciences IJCMS 4 (6) (June).

[14] Mohammed Javed, P., Nagabhusha, Chaudhuri, B. B. Extraction of Line Word Character Segments Directly from Run Length Compressed Printed Text Documents.

[15] Vassilopoulosa, Nikos., ErginaKavallieratoua. (2013). A Classification-free Word-Spotting System", SPIE-IS\&T, Vol. 8658, 86580.

[16] Manmatha, R., Han, Chengfeng., Riseman, E. M. Word Spotting: A New Approach to Indexing Handwriting, In: Proceedings of IEEE Computer Society Conference on Computer Vision and Pattern Recognition, CVPR '96, 1996, p. $631-637$.

[17] Park, Sang Cheol., Son, Hwa Jeong., Jeong, Chang Bu., Kim, Soo Hyung. (2005). Keyword Spotting on Hangul Document Images Using Two-Level Image-to-Image Matching, Springer-Verlag Berlin Heidelberg, p. 79-81.

[18] Maurya, Samudra Gupt., Chugh, Ritika., Rohit Kumar, Manoj, P, V. (2014). Capital Letter Recognition in Non Cursive Handwritten Documents, International Journal of Computer Science and Information Technologies (IJCSIT), $5(3)$.

[19] Sarkar, Sayantan. (2013). Word Spotting in Cursive Handwritten Documents using Modified Character Shape Codes. (http://arxiv.org/ftp/arxiv/papers/1310/ 1310.6063.pdf)

[20] Toni, M., Rath, Manmath, R. (2002). Word Image Matching Using Dynamic Time Warping, (January) (http:/ /ciir-publications.cs.umass.edu/pdf/MM-38.pdf

[21] Lu, Yue., Chew Lim Tan. (2002). Word Searching in Document Images Using Word Portion Matching", Springer p. 319-328.

[22] Nagendar, G., Jawahar, C. V. Efficient Word Image Retrieval using Fast DTW Distance Centre for Visual Information Technology, IIIT Hyderabad.

[23] Vijayarani, S., Sakila, A. (2016). A Survey on Word 
Spotting Techniques for Document Image Retrieval, International Journal of Engineering Applied Sciences and Technology, 1 (8).

[24] Mohammed Javed, Nagabhushan, P., Chaudhuri, B. B.Extraction of Line Word Character Segments Directly from Run Length Compressed Printed Text Documents, Research Gate.

[25] Roya, Partha Pratim Bhunia, Ayan Kumar., Ayan Das, Dhar, Prithviraj., Pal, Umapada. (2017). Keyword spotting in doctor's handwriting on medical prescriptions, Expert Systems With Applications, (January).

[26] O'Gorman, Lawrence., Kasturi, Rangachar (2009). Document Image Analysi, IEEE Computer Society Executive Briefings, 2009.
[27] Angelos, P., Giotis, Sfikas, Giorgos., Gatos, Basilis., Nikou, Christophoros. (2017). A survey of document image word spotting techniques, Pattern Recognition, 68, 310332.

[28] Kumar, Gaurav., Govindaraju, Venu., Bayesian. (2017) background models for keyword spotting in handwritten documents, Pattern Recognition, 64, 84-91.

[29] Angelos, P., Giotis, Giorgos Sfikas, Basilis Gatos, Christophoros Nikou, (2017). A survey of document image word spotting techniques, Pattern Recognition, 68, 2017.

[30] Zagoris, K., Pratikakis, I., Gatos, B. (2017). Unsupervised Word Spotting in Historical Handwritten Document Images Using Document-Oriented Local Features, IEEE Transactions on Image Processing, 26 (8) 4032-4041, (August). 